\title{
OPACIDAD REFERENCIAL Y ATRIBUCIÓN INTENCIONAL A ANIMALES SIN LENGUAJE
}

\author{
LAURA DANÓN
}

\begin{abstract}
In this paper I examine Davidson's argument from referential opacity against the attribution of thoughts to non-linguistics animals. I will begin by reconstructing the strongest version of the argument - i.e., the one which is better suited to overcome the different objections that have been raised against it. Once that is done, I will also object this version arguing, in a nutshell, that the fact that non-human animals lack language does not preclude us from acquiring some knowledge (although such knowledge may be partial) of their mental contents. Thus, I conclude that the argument from referential opacity fails to support Davidson's radical skepticism regarding the possibility of acquiring knowledge of the mental contents of non-linguistic animals, and of legitimately attributing intentional mental states to them.
\end{abstract}

Keywords: Animal minds; referential opacity; mental content; Davidson; intentional attribution.

\section{Introducción}

Donald Davidson $(1975 ; 1982 ; 1997)$ se cuenta entre los filósofos que han cuestionado con mayor vigor la legitimidad de atribuir estados mentales a criaturas sin lenguaje. Para apuntalar tal posición, este autor ofrece una serie de argumentos que, considerados en conjunto, constituyen uno de los ataques contemporáneos más influyentes a la tesis de que los animales poseen estados mentales intencionales. Aunque, a menudo, las objeciones de Davidson en contra del pensamiento animal se solapan de manera considerable, es posible, siquiera con fines de lograr mayor claridad expositiva, deslindar los siguientes argumentos: el de la opacidad referencial, el del holismo, el de la relación entre pensamientos y conceptos, y el de la dependencia del pensamiento respecto de la noción de verdad (Glock 2000; 2003).

En este trabajo examinaré exclusivamente el argumento de la opacidad referencial. En un primer momento, distinguiré varias interpretaciones posibles del mismo y presentaré algunas objeciones que se han dado en contra de unas u otras. Esto me permitirá identificar cuál es la versión más fuerte del argumento, esto es, aquella que está mejor posicionada para sortear las diversas objeciones presentes en la literatura. Dejando de lado preocupaciones exegéticas, en un segundo momento, me focalizaré en esta versión fuerte e intentaré disputar su alcance mostrando que, aún si el argumento de la opacidad logra establecer lo que pretende, tenemos razones pa-

Principia 20(2): 143-164 (2016).

Published by NEL — Epistemology and Logic Research Group, Federal University of Santa Catarina (UFSC), Brazil. 
ra ser (al menos relativamente) optimistas con respecto a la posibilidad de realizar atribuciones mentalistas legítimas a animales no humanos.

\section{El argumento de la opacidad referencial}

A grandes rasgos, el argumento de la opacidad referencial apunta a establecer que, en ausencia de lenguaje, resulta imposible especificar con el grado de precisión y "fineza de grano" necesarios el contenido de los estados mentales que atribuimos a una criatura. Para establecer tal conclusión, Davidson parte de dos afirmaciones poco controvertidas:

a) Las actitudes proposicionales poseen siempre algún contenido intencional, en tanto que refieren o tratan acerca de algo.

b) Los estados mentales representan de cierto modo o bajo cierto aspecto tal referente. Lo cual equivale a afirmar que dos contenidos mentales pueden tener un mismo referente pero, pese a ello, poseer contenidos diferentes que describan a este referente bajo distintos aspectos. Dicho de otro modo, los contenidos mentales son de grano más fino que los estados de cosas a los cuales refieren (Lurz 2008; Searle 1992; Crane 2013).

La aspectualidad o forma aspectual de nuestros contenidos mentales da lugar, a su vez, a una peculiaridad semántica de los enunciados que involucran términos mentales: su opacidad referencial o intensionalidad. Por lo general, cuando reemplazamos en un enunciado una expresión por otra que posee el mismo referente, preservamos el valor de verdad del enunciado original. Esta propiedad se quiebra, sin embargo, cuando los términos mentales hacen su aparición. En tales casos puede ocurrir que, si sustituimos dentro de un enunciado una expresión por otra co-referencial, obtengamos un nuevo enunciado con un valor de verdad diferente al del original. En otros términos: se quiebra el Principio de Sustitución, que estipula que si dos expresiones son co-referenciales, podemos reemplazarlas dentro de un enunciado sin alterar su valor de verdad. Suele señalarse, además, que en estos casos la opacidad referencial se debe a que las atribuciones de estados mentales procuran capturar la aspectualidad de los estados atribuidos, esto es, el modo específico en que el sujeto pensante se representa cierto objeto o estado de cosas (Lurz 2008; Searle 2006).

Ahora bien, según Davidson, la intensionalidad u opacidad referencial de las atribuciones mentalistas comporta un problema insuperable para quien pretenda atribuir estados mentales intencionales a los animales no lingüísticos. Esto se debe, arguye, a que el repertorio conductual de tales criaturas resulta excesivamente tosco y pobre para permitirnos distinguir qué contenido mental - entre una serie de contenidos co-referenciales alternativos - corresponde atribuirles y cuáles no. El perro mueve 
la cola y ladra cuando el dueño de casa abre la puerta. ¿Cree que su dueño ha llegado a casa? ¿Cree que ha llegado el gerente del Banco Nación? ¿O que quien ha entrado es el hombre que acostumbra tomar un café en el centro todas las mañanas?

El problema es que no podemos sencillamente atribuirle todos estos contenidos co-referenciales, o cualquiera de ellos de modo indistinto, pues:

I. Debido a la aspectualidad del contenido, si el perro tiene estados mentales acerca de su dueño, estos contenidos representan al dueño de un modo específico y no de otros.

II. Debido a la opacidad referencial, aunque algunas de estas atribuciones - las que capturan el aspecto específico bajo el cual fue pensado el referente resultarán verdaderas, otras, en cambio, serán falsas.

El problema que se nos plantea es que, en tanto carece de lenguaje, nada que el animal pueda hacer nos brinda evidencia lo suficientemente rica y precisa como para elegir, de un modo justificado, entre estas distintas atribuciones:

... a menos que haya conducta que pueda interpretarse como habla, la evidencia no resultará adecuada para justificar las distinciones finas que estamos acostumbrados a hacer en la atribución de pensamientos. Si persistimos en atribuir deseos, creencia u otras actitudes bajo esas condiciones, nuestras atribuciones y consecuentes explicaciones de acciones se verán seriamente indeterminadas por cuanto muchos sistemas alternativos de atribución, muchas explicaciones alternativas, estarán igualmente justificados por los datos disponibles. (Davidson 1975, p.172).

Luego, aunque necesitamos determinar qué contenidos atribuir al animal, su tosco comportamiento imposibilita la tarea. Se puede tratar de sortear esta dificultad apelando a la distinción entre dos tipos diferentes de atribuciones doxásticas: las atribuciones de dicto y las de re (Quine 1956). Al atribuir a una criatura una creencia de dicto, la estamos comprometiendo con la aceptación de un dictum particular, esto es, con la aceptación de cierta descripción del referente de su pensamiento. Las adscripciones de re, en cambio, no procuran capturar bajo qué descripción representa la criatura aquello en lo que piensa, sino simplemente identificar cuál es dicho referente. De allí que, mientras las atribuciones de dicto son opacas, las atribuciones de re son transparentes, admitiendo la sustitución de términos co-refenciales sin cambiar su valor de verdad.

Haciendo uso de esta distinción cabe argüir que, aún concediendo que el argumento de la opacidad semántica permita cuestionar la legitimidad de nuestras atribuciones de creencias de dicto a los animales no humanos, este no basta para minar nuestras atribuciones de re, en tanto las últimas son referencialmente transparentes. Luego, aunque no podamos decir del perro que escarba el suelo que cree que hay 
un hueso escondido bajo tierra - pues nos resulta imposible determinar, en ausencia del lenguaje, bajo qué aspectos captura tal objeto - sí podemos decir que cree del hueso (sin importar con qué rasgos se lo represente) que está bajo tierra. La intención de nuestra atribución, en este caso, es simplemente indicar cuál es el objeto al cual refiere la creencia del animal, sin comprometernos por ello con un modo específico en que él se represente dicho objeto. Si bien nos brindan información más tosca y limitada, estas atribuciones transparentes poseen la virtud de permitirnos referir de modo laxo a las creencias de una criatura, aún cuando no conozcamos su contenido preciso, como parece ocurrir en el caso de los animales no humanos.

Ahora bien, Davidson (1982) ha objetado explícitamente esta línea argumentativa. Según señala, aún cuando las construcciones de re puedan relevar al intérprete de la tarea de hallar aquella descripción particular que captura el contenido de la creencia del animal, no modifican el siguiente hecho fundamental: si el animal en cuestión tiene una creencia, tal descripción debe existir. Dicho en otros términos: dado el carácter aspectual de nuestros estados mentales intencionales, si el animal cree algo acerca de $\mathrm{X}$, ha de creerlo bajo alguna descripción precisa de X y no bajo otras.

\begin{abstract}
Alguien podría pensar que la posición que ocupa 'ese roble' dentro de la oración: 'El perro piensa que el gato ha subido a ese roble' es transparente (dicho en la terminología de Quine). La manera correcta de exponer la creencia del perro - continúa la propuesta - es 'El perro piensa con respecto a ese roble, que el gato ha subido a él' o, 'Ése es el roble al que el perro piensa que ha subido el gato'. Pero tales construcciones, aunque liberan a quien hace la atribución de la necesidad de producir una descripción del objeto que aceptase el sujeto de la creencia, implican sin embargo que hay alguna descripción así; la descripción de re identifica un objeto que el sujeto de la creencia podría identificar de alguna manera. (Davidson 1982, p.145)
\end{abstract}

Hasta aquí me he limitado a reconstruir los aspectos centrales del argumento davidsoneano. Quedan aún, sin embargo, algunos puntos interpretativos problemáticos por dirimir. Como vimos, el argumento bajo examen trata de establecer que, debido a la opacidad referencial de nuestras atribuciones de estados mentales, sólo podemos atribuir estados mentales intencionales a aquellas criaturas que poseen un lenguaje. Un primer interrogante que surge, llegado este punto, es el siguiente: ¿este argumento pretende establecer que el dominio de un lenguaje constituye sólo una condición necesaria o, de modo más ambicioso, una condición necesaria y suficiente para tales atribuciones? Independientemente de cuál sea la opción que corresponda atribuir a Davidson desde un punto de vista estrictamente exegético, hay buenas razones para sostener que la versión más defendible del argumento es aquella que sólo considera la posesión de lenguaje como condición necesaria - aunque no suficiente - para atribuir a una criatura $\mathrm{C}$ contenidos mentales específicos. Para ver esto, partamos de

Principia 20(2): 143-164 (2016). 
la siguiente observación poco controvertida: C puede dominar un lenguaje y realizar emisiones sinceras sobre el contenido de sus creencias que, sin embargo, no siempre brindarán evidencia suficiente para determinar cuáles son exactamente los contenidos de sus pensamientos. Esto es lo que ocurre, por ejemplo, cuando lo que alguien dice en un momento no se condice con otras afirmaciones previas o posteriores suyas, cuando lo que dice entra en clara contradicción con lo que hace, o con lo que debería creer dados los otros estados mentales que posee, el contexto en el que se encuentra, sus experiencias previas, etc.

De modo semejante, especificar cuáles son los contenidos de los pensamientos de un sujeto se torna una tarea de dudosa resolución cuando, por ejemplo, este dice creer algo acerca de un objeto $\mathrm{O}$, pero a la vez revela poseer ciertas creencias bizarras sobre $\mathrm{O}$, o ignora información relevante acerca de $\mathrm{O}$, llevándonos a dudar si realmente lo que él está pensando queda adecuadamente capturado atribuyéndole el concepto O. Siguiendo a Wilkes (1997), pensemos en el caso en que atribuimos a un sujeto ordinario una creencia cualquiera acerca del estado de sus huesos. Este sujeto, sin embargo, desconoce las diferencias entre los animales vertebrados e invertebrados y, por lo tanto, no sabe que los huesos forman parte del esqueleto de los animales vertebrados. O pensemos en el anatomista que posee la información anterior, pero tiene, pese a ello, algunas creencias bizarras acerca de los huesos humanos, que no armonizan con el resto de nuestra concepción bioquímica de los huesos (él puede pensar, por ejemplo, que los huesos humanos contienen una sustancia espiritual). En todos estos casos, las emisiones lingüísticas de $\mathrm{C}$ sugieren que posee cierto estado mental con un contenido aspectual específico, pero hay otras buenas razones - que pueden incluso ser más poderosas que las que brinda la evidencia lingüística puntual - para no atribuirle dicho estado.

Shwitzgebel (2001) ha acuñado un término específico - tener un estado "de creencia intermedio" (in-between believing) — para agrupar aquellos fenómenos, que él considera prevalentes, en los que un sujeto muestra un patrón de disposiciones conductuales fluctuantes y dispares, algunas de las cuales sugieren que, efectivamente, cree en una proposición P, mientras que otras indican lo contrario. En todos ellos, conocer la conducta lingüística de un sujeto resulta insuficiente para establecer con precisión qué es lo que cree. Esto puede deberse a que algunas de las cosas que el sujeto dice apuntan en la dirección de atribuirle cierto contenido P, mientras que otras nos guían en dirección opuesta; o puede deberse a que, mientras la conducta lingüística sugiere que el sujeto cree que $\mathrm{P}$, hay otros comportamientos no lingüísticos que indican algo diferente.

Si se toman en cuenta los fenómenos señalados cabe concluir que, para que podamos conocer con precisión el contenido específico de los pensamientos de una criatura, no basta con que esta sea lingüísticamente competente, sino que, como el mismo Davidson parece reconocer en su teoría de la atribución intencional, deben 
preservarse vínculos de coherencia y consistencia global entre lo que este afirma sobre sus estados mentales, las acciones que realiza, el contexto en el que se encuentra y otros estados mentales potencialmente vinculados a aquel que deseamos atribuirle. En la medida en que tales condiciones no se cumplan, enfrentaremos dificultades crecientes para especificar sus contenidos mentales, que no son tan distintas de las que nos asolan en el caso de las atribuciones a los animales no humanos. Aceptemos, pues, que de acuerdo con la lectura más caritativa que podemos hacer del mismo, el argumento de la opacidad referencial sólo sostiene que la competencia lingüística de una criatura es condición necesaria para poder establecer con precisión cuáles son los contenidos de sus estados mentales intencionales.

Otro nodo de debate surge en torno a cuál es la conclusión que el argumento intenta establecer. En ocasiones, Davidson parece sostener que, en tanto una criatura no muestre un patrón de comportamientos tan rico como el que poseen quienes dominan un lenguaje, no sólo careceremos de justificación para atribuirle pensamientos con contenidos intencionales, sino que deberemos concluir también que, de hecho, carece de tales pensamientos (Searle 1994; McGinn 1982; Davidson 1982).

Bajo esta versión ambiciosa, el argumento davidsoneano resulta fácilmente objetable. Como hemos visto, éste parte de señalar que en ausencia de lenguaje la conducta del agente interpretado resulta demasiado tosca para permitirnos dirimir entre distintos contenidos alternativos, aspectualmente diferentes, cuál cabe atribuirle. Ahora bien, hasta aquí parece que, en el mejor de los casos, lo que el argumento logra es establecer un punto epistemológico: que en ausencia de lenguaje no podemos justificar nuestras atribuciones intencionales a animales no humanos. Pero de allí no se sigue que tales criaturas de hecho carezcan de pensamientos (Searle 1994; Heil 1992; Schwitzgebel 1997). Las razones para criticar este paso adicional son al menos dos. Por una parte, cabe aducir que de un enunciado epistémico del tipo "no tenemos buenas razones para creer que P" no se sigue una conclusión ontológica del tipo: "no es el caso que P". Por otra parte, podemos objetarlo porque de la afirmación epistemológica según la cual "no estamos justificados en atribuir al animal ninguna creencia particular" no se sigue que no tengamos justificación para afirmar que el animal tiene creencias en general (aún cuando no podamos afirmar qué creencias particulares posee) (Schwitzgebel 1997). Parece, pues, que la versión más sólida del argumento de la opacidad es aquella que apunta meramente a establecer una conclusión epistemológica: en ausencia de lenguaje no podemos llegar a saber si una criatura tiene estados mentales intencionales dotados de contenidos, por la simple razón de que no podemos saber cuáles son tales contenidos.

Ahora bien, aunque la tarea sea más laboriosa, también podemos cuestionar esta versión modesta, mostrando que la carencia de lenguaje por parte de los animales no humanos no constituye un impedimento decisivo a la hora de adquirir conocimiento — siquiera parcial — sobre los contenidos de sus estados mentales. En otras palabras: 
aún si en ausencia de lenguaje fuera, como Davidson pretende, imposible obtener el tipo de evidencia requerida para justificar la atribución de ciertos contenidos, especificados con toda precisión y en su grano más fino, esto no bastaría para concluir que no tenemos acceso epistémico alguno a los mismos. Por el contrario, según intentaré mostrar, podemos saber: que una criatura tiene estados mentales dotados de contenidos aspectuales aún cuando no sepamos exactamente cuáles son tales contenidos, que algunas de nuestras adscripciones lingüísticas capturan mejor que otras tales contenidos (aún cuando no lo hagan de modo perfecto) y que, sea cual sea el contenido exacto de un estado mental, si ciertas atribuciones capturan mejor que otras cierto contenido es porque este se asemeja a las primeras más de lo que se asemeja a las segundas. De todo esto se sigue, claramente, que tenemos algún conocimiento relevante sobre la forma aspectual de tales contenidos. El argumento de la opacidad referencial no parece, por tanto, dar sustento al escepticismo radical davidsoniano respecto de la posibilidad de conocer los estados mentales de los animales carentes de lenguaje.

\section{El problema de la aspectualidad y el conocimiento de los contenidos mentales de los animales}

Aceptemos, apoyándonos en las consideraciones del apartado anterior, que la variante más plausible del argumento de la opacidad referencial es aquella que apunta a establecer una conclusión epistemológica con respecto a nuestra imposibilidad de conocer cuáles son los estados mentales intencionales de los animales sin lenguaje. Aceptamos también, por mor del argumento, que cuando una criatura carece de lenguaje su conducta se torna demasiado tosca como para permitirnos dirimir qué contenido específico tienen los estados mentales que subyacen a la misma. En lo que sigue querría mostrar que esto no nos impide tener cierto conocimiento, aún cuando este sea tosco y parcial, de los contenidos de dichos estados mentales. Y tal conocimiento, pese a sus límites, puede resultar suficiente para legitimar nuestras atribuciones mentalistas a tales criaturas.

Permítaseme comenzar por deslindar de qué índole es el conocimiento parcial de los estados mentales de los animales que, pienso, podemos obtener. Recordemos, a tal fin, los puntos a) y b) señalados en la sección anterior: los contenidos mentales se caracterizan por tener un referente - aquello acerca de lo cual versan - y una forma aspectual - el modo o aspecto bajo el cual se presenta tal referente. Apoyándonos en esta distinción introductoria, podemos distinguir, al menos, tres aspectos o dimensiones diferentes del contenido que nos interesa conocer: i) cuál es su referente; ii) si dicho referente es pensado bajo una forma aspectual entre otras posibles; iii) cuál es la forma aspectual en cuestión. 
Un supuesto adicional subyace a la discusión que sigue y, espero, ha de ganar plausibilidad a partir de la misma: el conocimiento de un estado mental intencional no tiene por qué ser una cuestión de "todo o nada", sino que puede presentarse en distintas formas y grados. Esta idea general puede, a su vez, descomponerse en dos afirmaciones más específicas. La primera de ellas es que podemos conocer algunos de los aspectos identificados anteriormente (i-iii) aunque no conozcamos otros. La segunda es que podemos tener capacidades más o menos robustas para conocer cada una de estas dimensiones y un conocimiento más o menos rico, detallado y/o preciso de las mismas. Trazadas ya estas distinciones previas veamos, en las próximas subsecciones, qué se puede decir acerca de las dimensiones identificadas en i-iii).

\subsection{El conocimiento del referente de un estado mental}

Querría comenzar aquí por mostrar que, en ocasiones, podemos conocer cuáles son los referentes de los estados mentales de un animal aún cuando no sepamos bajo qué aspecto se representa a tal referente. Algo que, por su parte, el mismo Davidson parece conceder cuando admite que es posible realizar atribuciones de re a los animales no humanos (aunque luego proceda a objetarlas sobre la base de que presuponen una descripción bajo la cual el animal en cuestión está capturando al referente).

Cuando atribuimos un estado mental a un animal lo hacemos basándonos en evidencia conductual. Ahora bien, usualmente estos comportamientos tienen una direccionalidad o un "objetivo externo": apuntan a la realización u obtención de cierto objeto o estado de cosas, del mismo modo en que una flecha apunta a una diana (Tomasello et al. 2005; Lurz \& Krachun 2011). Los objetivos externos han de distinguirse, en dos sentidos, de los "objetivos internos", entendidos como estados mentales, intencionales y conativos, que guían la conducta del agente. Por una parte, es posible que ciertas entidades o sistemas tengan objetivos externos aunque carezcan de objetivos internos. Este sería, claramente, el caso del misil que se dirige a su objeto, de la pelota que se dirige al arco, etc. Por otra parte, también es posible que, en tanto intérpretes, captemos los objetivos externos de una conducta con independencia de que seamos capaces de atribuir a quien la realiza objetivos internos (o, de modo más general, con independencia de que seamos capaces de atribuirle estados mentales en absoluto).

Partiendo de estas distinciones, querría comenzar por defender aquí que, a menudo, los intérpretes podemos captar la direccionalidad o el objetivo externo de la conducta de un agente, en el mismo sentido no mentalista en el que podemos identificar que una flecha se direcciona a la diana, aún cuando no sepamos nada acerca de los estados mentales del agente. Y esta direccionalidad básica del comportamiento nos brinda un primer indicador y una primera puerta de acceso (falible pero útil) al conocimiento de ciertos estados mentales simples, como pueden ser algunos deseos

Principia 20(2): 143-164 (2016). 
o intenciones del animal: nos ayuda a identificar a su referente. Dicho de otro modo: la posibilidad de identificar objetivos externos opera como una buena guía hacia la identificación del referente tanto de los objetivos internos, como de otros estados conativos de la criatura. ${ }^{1}$

Antes de proseguir querría, sin embargo, detenerme a examinar una posible objeción. Alguien podría aducir que, para seguir la estrategia argumentativa que propongo, es preciso asumir precisamente aquello que estaba en cuestión. A saber: que es posible y legítimo explicar ciertos comportamientos no lingüísticos de una criatura atribuyéndole estados mentales intencionales antes de haber dirimido si, efectivamente, satisfacen el requisito de aspectualidad y dan lugar a adscripciones referencialmente opacas. Permítaseme, pues, detenerme en este punto. A mi entender, la aspectualidad y la posibilidad de dar lugar a adscripciones referencialmente opacas son rasgos centrales que los estados mentales intencionales deben satisfacer. Pero no son los únicos requisitos relevantes. Por el contrario, hay muchos otros, tales como: la flexibilidad conductual, la posibilidad de realizar inferencias, la capacidad para distanciarse de los estímulos que impactan sobre la criatura en su aquí-y-ahora, la normatividad, etc. ${ }^{2}$ Luego, pienso, cuando queremos dar cuenta del comportamiento de una criatura podemos partir de preguntarnos si hay buenos indicadores de que ésta cumple con algunos otros de los requisitos asociados a la posesión de estados mentales intencionales. Si este es el caso, tendremos algunas razones para atribuirle - tentativamente - algunos estados mentales intencionales. Posteriormente, podremos proceder a examinar si podemos detectar el referente de sus presuntos pensamientos, si estos hipotéticos estados mentales poseen una forma aspectual y, finalmente, podremos tratar de averiguar cuanto podamos acerca de la misma. En la medida en que logremos avanzar con éxito en cada uno de estos puntos, se verán reforzadas las razones iniciales para pensar que la criatura bajo examen posee efectivamente tales estados intencionales.

Volvamos ahora a examinar cómo puede tener lugar la identificación de los referentes de ciertos estados mentales simples, como percepciones y deseos, que (presuntamente) guían la conducta de algunos animales no humanos. Retomemos, nuevamente, el caso del perro que escarba bajo la tierra, allí donde el día anterior lo vimos enterrar un hueso. Parece sensato hipotetizar que lo que está tratando de desenterrar es un hueso, por dos razones. En primer lugar, porque su conducta se dirige hacia el hallazgo del hueso. La idea aquí, como indiqué anteriormente, es que los intérpretes podemos entender a qué apunta una conducta, o cual es su objetivo externo, y esta direccionalidad básica del comportamiento nos brinda un primer indicador y una primera puerta de acceso (falible pero útil) al conocimiento del deseo, o la intención, del animal: nos ayuda a identificar a su referente. En segundo lugar, nuestra identificación del hueso como el referente del deseo que anima la conducta del perro encontrará un sustento adicional si, posteriormente, este da señales de satisfacción 
y cesa la conducta de búsqueda al desenterrar el hueso.

Generalizando y sintetizando: para identificar el referente de intenciones o deseos, al menos en los casos más básicos, puede resultar suficiente apoyarnos en dos tipos de comportamientos. Por una parte, en los comportamientos de agente dirigidos a obtener un cierto objeto o estado de cosas y, por otra, en el cese de dichos comportamientos cuando el objeto o estado de cosas deseado ha sido alcanzado. ${ }^{3}$

Por otra parte, parece que cuando hemos de identificar el referente de un estado de percepción, son otros los comportamientos y datos observables que resultan relevantes. En el caso de un estado de percepción visual será importante tomar en consideración si hay una línea directa que vaya desde la mirada del sujeto a algún objeto $\mathrm{O}$, o hacia algún estado de cosas E. Adicionalmente, también será relevante detectar si el sujeto interpretado se involucra en algún comportamiento direccionado hacia objeto O. Si contamos con evidencia de alguno de estos dos tipos (o, en el mejor de los casos, si contamos simultáneamente con evidencia de ambas clases), tenderemos a atribuir al agente interpretado un estado perceptual acerca de $\mathrm{O}$.

Llegado este punto, me parece importante señalar explícitamente cuán modesta es la tesis que defiendo. Según vimos, en ocasiones, resulta poco problemático detectar cuál es el referente de un ejemplar particular de estado mental. Este parece ser, por ejemplo, el caso de la instanciación específica del deseo de recuperar el hueso que tiene el perro del ejemplo arriba empleado. Sin embargo, esto no basta para garantizar que el concepto bajo el cual dicho animal subsume a los huesos en distintas ocasiones tenga la misma extensión que nuestro concepto de hueso. La razón de ello es la siguiente: determinar cuál es la extensión del concepto de $x$ que posee una criatura $C$ requiere saber qué caería bajo la extensión de $x$ en distintas situaciones contrafácticas. ${ }^{4}$ Lo cual, a su vez, parece exigir que investiguemos cómo aplica C el concepto de $x$ a distintos particulares en las más variadas situaciones, y no sólo determinar a qué está respondiendo en esta situación particular.

Ahora bien, aún si tomamos en consideración estas limitaciones, sigue habiendo una asimetría notable entre el acceso epistémico que tenemos al referente de una instanciación particular de un estado mental y el acceso que tenemos a la forma aspectual de su contenido. En el primer caso, podemos saber a qué refiere este estado mental en esta situación, basándonos en indicadores conductuales o ambientales observables, que pueden ser enteramente no-lingüísticos. Sin embargo, esta misma evidencia parece insuficiente para establecer con precisión bajo qué aspecto se representa la criatura dicho referente, incluso en el caso de una instanciación particular de un pensamiento. Así, podemos establecer que la instancia de deseo que anima la conducta de mi perro es acerca del hueso, sin saber por ello de qué modo se representa al hueso - qué conjunto específico de notas o rasgos le adscribe - ni siquiera en este momento y situación particular. Todo lo cual nos indica que el problema del acceso epistémico a la aspectualidad del contenido merece un tratamiento diferente. 


\subsection{El conocimiento del carácter aspectual de un estado mental}

Hasta aquí he argumentado que, al menos en algunas ocasiones, podemos identificar el referente de los ejemplares de estados mentales de una criatura carente de lenguaje. Esto basta para garantizar que no estamos conminados a la ignorancia absoluta sobre los contenidos mentales de los animales no humanos. Pero nuestra posibilidad de avanzar en la delimitación de cuáles sean los contenidos mentales atribuibles a tales criaturas no se agota ahí. Según señalé, hay al menos dos pasos adicionales que podemos dar.

He aquí el primero de ellos: podemos determinar bajo qué condiciones es legítimo atribuir a un animal estados mentales cuyos contenidos poseen una forma aspectual, aún cuando seamos incapaces de precisar cuál es esa forma aspectual en cada caso particular. Dicho de otro modo: podemos establecer que una criatura efectivamente tiene un pensamiento acerca de un referente $\mathrm{X}$, representando a $\mathrm{X}$ bajo cierto aspecto, aún cuando no sepamos exactamente cuál es este último. ${ }^{5}$

Partamos de recordar que es usual explicar la opacidad referencial de las atribuciones de estados mentales aduciendo que éstas están capturando el modo específico en que una criatura representa un referente. Parece, pues, que un buen camino para establecer que una criatura tiene pensamientos aspectuales, consistirá en mostrar que despliega patrones conductuales lo suficientemente ricos y complejos como para que, al tratar de explicarlos apelando a atribuciones intencionales, nos encontremos con que los contenidos de dicha atribución quiebran el Principio de Sustitución (en adelante PS) de un modo no trivial. Esto daría buenas razones para pensar que la criatura en cuestión es capaz de formarse representaciones aspectuales de dichos referentes.

Comencemos, pues, por identificar de modo general qué tipo de patrones conductuales son aquellos que, de ser explicados en términos intencionales, darían lugar a adscripciones referencialmente opacas, que quiebren PS de modo robusto. Para alcanzar este objetivo examinaré dos casos hipotéticos de criaturas con capacidades, de diverso grado de complejidad, para actuar en relación con un objeto de su entorno. Según veremos, sólo el segundo tipo de criaturas muestra el tipo de conductas que estamos buscando.

a) Los "meros reactores"

Imaginemos, en primera instancia, un hipotético animal A, de conducta tan simple y estereotipada que sólo puede dar una única respuesta conductual $C_{1}$ ante cierto objeto $\mathrm{O}$. Pensemos, además, que A es incapaz de aprender nada nuevo acerca de $\mathrm{O}$, con lo cual su respuesta ante $O$ permanece rígida e inalterable a lo largo del tiempo. Este es el tipo de criatura que Duhau (2010) ha llamado un "mero reactor".

Según vimos, si los pensamientos de una criatura tienen contenidos dotados de

Principia 20(2): 143-164 (2016). 
una forma aspectual, al ponerlos en palabras estos han de quebrar PS. Ahora bien, alguien podría aducir que, aún en el caso de criaturas de conductas tan rígidas como las de los meros reactores, basta con que nosotros qua intérpretes les atribuyamos otras representaciones co-referenciales diferentes de las que poseen para que se quiebre PS. Pues si X sólo puede representar el objeto O bajo un aspecto, al atribuirle cualquier representación alternativa de $\mathrm{O}$ pasaremos de una atribución verdadera a una falsa.

Para ilustrar esta idea, supongamos que el sapo que saca la lengua ante cada mosca que pasa es un mero reactor. ${ }^{6}$ Asumamos, adicionalmente, que cabe atribuirle una creencia con un contenido aspectual determinado acerca de la mosca. Esta creencia podría ser, por ejemplo, la de que algo comestible se acerca a él. Luego, podría parecer que basta con que qua intérpretes reemplacemos la atribución de que el sapo cree que algo comestible se acerca por otra co-referencial - como que él cree que algo peligroso se acerca - para pasar de un enunciado verdadero a uno falso.

Pienso, sin embargo, que en estos casos PS no se quiebra de modo genuino. La razón es la siguiente: en tanto nuestra criatura $\mathrm{X}$ sólo es capaz de representar a $\mathrm{O}$ bajo un único aspecto, al reemplazar una atribución intencional que capture dicho contenido por la atribución de cualquier contenido co-referencial alternativo no estamos realizando atribuciones posiblemente verdaderas pero falsas, sino adjudicándole contenidos carentes de sentido para ella. ${ }^{7}$ Ahora bien, según lo indica explícitamente la definición de PS, un genuino quiebre de este principio tiene lugar cuando es posible que, al cambiar un modo de representar al referente por otro, pasemos de una atribución verdadera a una falsa (lo cual no es lo mismo que pasar a un sinsentido). Esto nos conduce a concluir que, para que nuestras atribuciones de estados mentales con contenidos aspectuales estén justificadas, han de cumplir con requisitos más exigentes de lo que podría parecer en primera instancia.

b) La criatura que representa el mismo referente bajo distintos aspectos

El tipo de casos examinados en a) nos indica que, para que sea posible que nuestras atribuciones intencionales a una criatura quiebren PS de modo genuino, necesitamos que ésta cuente con más de un modo de representar un mismo referente. Ahora bien, ¿cuáles son los comportamientos que indican que esto ocurre?

Para responder a esta pregunta apelemos a un segundo caso hipotético: pensemos en un animal A que responde, en momentos distintos, de dos modos claramente diferentes ante un mismo objeto. En un primer momento, por ejemplo, A sale en busca de una nuez y cuando la encuentra la guarda un buen rato consigo hasta que se suscita una pelea, contexto agonístico en el cual la arroja en contra de un enemigo. En otro momento, A sale en busca de una nuez pero, cuando la encuentra, la casca y se la come.

Asumamos ahora - por mor del argumento — que estas conductas están guia-

Principia 20(2): 143-164 (2016). 
das por algún tipo de estado mental. Admitamos, además, que parte de lo que nos permite individuar a un estado mental es su papel cognitivo o funcional, el cual se encuentra constituido, en parte, por los vínculos causales entre dicho estado mental y la conducta. Luego, dada la disparidad de las conductas que se producen en uno y otro caso, parece plausible hipotetizar que los estados mentales involucrados en ambos casos no son los mismos. Más aún, parece que, si queremos explicar sus comportamientos, tendremos que intentar, siquiera de modo tosco y grueso, capturar el modo en que tales contenidos representan la nuez en cada caso. Así, por ejemplo, podremos decir que, en uno de estos casos A se representa la nuez como algo semejante a un alimento comestible mientras que, en el otro, ve a la nuez como alguna suerte de arma que puede arrojar contra sus enemigos.

Pero, si este es el caso, bien puede ocurrir que al pasar de atribuirle a A la creencia de que tiene un arma que puede arrojar contra su enemigo a atribuirle la creencia de que tiene en su mano un alimento, se altere el valor de verdad de la atribución original, o que éste se conserve sin modificaciones. Basta para ello con que, qua intérpretes, equivoquemos la atribución y, por ejemplo, en el contexto en que A está por agarrar una nuez para comerla, le atribuyamos el deseo de obtener un arma para arrojarle a un enemigo o viceversa.

Llegado este punto es importante notar que, para que se satisfaga PS, la criatura $\mathrm{X}$ no necesita contar con un repertorio representacional tan rico y variado como el de los animales humanos. Sin duda, en la medida en que posea un espectro más nutrido de representaciones, podrá pensar acerca de un mismo referente bajo una mayor variedad de aspectos. Algo que, a su vez, posibilitará un mayor número de atribuciones intencionales, que resulten referencialmente opacas y no caigan en sinsentidos. Pero esta es, en último término, una diferencia puramente cuantitativa. Capacidades representacionales relativamente modestas, como las arriba señaladas, bastarán para dar lugar a (algunas pocas) atribuciones intencionales referencialmente opacas en un sentido robusto del término.

Recapitulando: tendremos buenas razones para atribuir a una criatura carente de lenguaje contenidos aspectuales diferentes acerca de un objeto $O$ siempre que en distintos momentos y contextos ésta se comporte, en relación con $\mathrm{O}$, de modos que sean claramente dispares entre sí y que merezcan explicaciones intencionales. La razón de ello es simple: a fin de dar las explicaciones intencionales requeridas para cada caso, tendremos que acudir a contenidos diferentes, que versen sobre distintos aspectos de $\mathrm{O}$, aunque no podamos precisar con exactitud cuáles sean estos (pues, de lo contrario, seremos incapaces de explicar la disparidad conductual de la criatura). Así pues, cada vez que estemos frente a una criatura que satisfaga estos requerimientos, se quebrará PS de modo genuino.

Principia 20(2): 143-164 (2016). 


\subsection{El conocimiento aproximado del contenido aspectual}

Supongamos que estamos ante un caso en el cual hemos logrado tener algún conocimiento - del tipo especificado en a) y b) — sobre algún estado mental particular de un animal. Esto es, asumamos que conocemos cuál es el referente de dicho estado mental y que sabemos, además, que la criatura en cuestión se representa este referente de un modo particular entre otros. La pregunta que se impone a continuación es la siguiente: ¿podemos acaso saber algo más acerca de la forma aspectual específica de este estado mental? En lo que sigue defenderé que, aún si Davidson está en lo correcto y no podemos conocer con precisión cuál es el contenido específico de dicho estado mental, dada la falta de lenguaje de su propietario, esto no nos impide tener algún conocimiento sustantivo, aunque imperfecto y parcial, no sólo del referente, sino también de la forma aspectual del mismo.

Para mostrar este punto, imaginemos a un mono que ha arrancado una fruta del árbol para comerla aún cuando, en otras ocasiones, la arranca para arrojarla a sus enemigos. Como ya señalé con anterioridad, el que el mono se muestre capaz de realizar dos conductas tan disímiles en relación con un mismo objeto parece exigirnos que, si queremos ofrecer una explicación intencional de sus comportamientos, le atribuyamos en cada caso estados mentales con contenidos representacionales diferentes. Más aún, en primera instancia se nos ocurren varias atribuciones alternativas posibles que podríamos proponer como candidatos a capturar cada uno de estos estados mentales. Así, podríamos caracterizar el contenido perceptual que guía el acto de arrancar la fruta y comerla empleando alguna de las siguiente alternativas: él percibe que ahí hay una fruta, él percibe que ahí hay alimento, él ha visto que ahí hay una banana, etc. De modo semejante, podríamos caracterizar el estado perceptual que guía el acto de arrojar la fruta como él ve la fruta como un arma, él ve la fruta como un proyectil arrojable, él ve la fruta como algo con lo cual golpear a su enemigo, etc. Llamemos a estos dos grupos, respectivamente, "1" y "2".

Parece preciso admitir que ninguna de las opciones que se nos ofrece en 1 y 2 logra capturar de modo preciso lo que piensa el mono en cada caso. Sin embargo, es claro que los contenidos seleccionados en 1 son muy diferentes de los seleccionados en 2 y que, en cambio, no se presentan relaciones de disimilitud tan marcadas como las que hay entre los integrantes de cada uno de estos grupos.

Luego, parece que contamos ya con un primer modo - grueso y tosco - de seleccionar entre mejores y peores modos de caracterizar los contenidos mentales de nuestro mono. Si este se come la fruta que ha arrancado, cualquiera de las atribuciones que pertenecen al grupo 1 será una mejor alternativa que cualquiera de las del grupo 2. Y si, en cambio, arroja el fruto como un arma, la relación será la inversa.

Esto basta para mostrarnos, pienso, que hay algo que sí podemos saber con respecto al modo específico en que nuestro chimpancé se representa la banana en cada 
caso: podemos saber que la representa de un modo que se asemeja a aquellos contenidos hipotéticos que hemos ubicado en el grupo 1 y que no la representa de un modo que se asemeje a los contenidos ubicados en el grupo 2 (o viceversa). Llegado este punto, sin embargo, alguien podría insistir en que aún queda pendiente la tarea de dar respuesta a la siguiente pregunta: ¿en qué aspectos se asemejan las atribuciones del grupo 1 al contenido que, de hecho, posee el animal? Lo cual, dado que todas las atribuciones que hemos ofrecido están formuladas, ineludiblemente, en nuestro lenguaje y empleando nuestros conceptos, equivale a preguntar: ¿en qué aspectos se asemeja la representación aspectual de la fruta que tiene el mono a aquella que queda capturada por nuestros conceptos de "fruta", "alimento" o "banana"? Cabe reconocer, además, que este interrogante no es de importancia menor. Pues identificar siquiera algunos puntos de semejanza entre los conceptos que figuran en nuestras atribuciones y las representaciones del animal parece indispensable para poder explicar por qué consideramos que estas son buenas aproximaciones a los contenidos mentales del animal y para avanzar en la especificación del modo en que éste se representa al fruto.

He aquí el modo en que, pienso, podemos avanzar en la respuesta al interrogante planteado. Tomemos la atribución que, a nuestro entender, es mejor candidata a capturar el contenido del estado mental que guía al mono a agarrar un fruto y comerlo. Supongamos que este reza: el percibe que allí hay una fruta. En este caso, tenemos buenas razones para sospechar, por ejemplo, que nuestro concepto fruta - el cual figura de modo ineludible en nuestra atribución - no es el mismo que el concepto de la fruta que posee el mono.

Siguiendo de cerca una propuesta realizada originariamente por Goldman (2006), en el contexto de un debate diferente, podemos lidiar con este tipo de casos diciendo algo como lo siguiente: el mono ha percibido una "fruta". En este caso, el uso de las comillas indica que nos abstenemos de emplear sin más nuestro concepto de fruta para replicar el del mono, porque hipotetizamos que hay diferencias relevantes entre ambos. Sin embargo, aún usamos dicho concepto, entrecomillado, para referirnos indirectamente, y quizás transitoriamente, al concepto que el mono posee, aún cuando no sepamos exactamente cuál sea este. ${ }^{8}$ Ahora bien - y lo que añado no está ya en Goldman - aunque no estemos tratando de emplear nuestro concepto de fruta para replicar el modo en que el mono se representa la fruta, no por ello es trivial o irrelevante que hayamos elegido entrecomillar precisamente este concepto de nuestro repertorio y no otros. Y no lo es, pienso, porque la elección del mismo descansa en una hipótesis implícita antes mencionada: la de que el concepto del mono es similar, al menos en parte, a nuestro concepto de fruta. En otras palabras: el concepto entrecomillado está allí para sugerirnos la existencia de similitudes entre el modo en que el mono está percibiendo la fruta y aquel en el que nosotros pensamos en ella cuando usamos el concepto de fruta, mientras que las comillas cumplen la función 
de indicar las diferencias que habría entre ambos casos.

Ahora bien, pese a estas aclaraciones sobre el carácter de nuestra atribución, sigue en pie la pregunta inicial: ¿es posible especificar mejor en qué se asemeja nuestro concepto de fruta al de "fruta" que hemos atribuido al mono? Pienso que podemos dar una respuesta afirmativa si partimos de la idea - cara a una extensa tradición que se remonta hasta Sellars (1963) - de que los contenidos mentales se encuentran constituidos por sus vínculos - caracterizados, dependiendo del autor, como funcionales, inferenciales, causales o conceptuales (Piccinini 2004) - con: i) ciertos estímulos externos; ii) otros estados mentales internos y iii) las respuestas conductuales del sujeto. ${ }^{9}$ Luego, lo que hace que un contenido $C_{1}$ sea el que es - y lo que, a su vez, lo diferencia de otro contenido $C_{2}$ - es qué estímulos externos o qué estados mentales hayan suscitado a $C_{1}$, qué interacciones mantenga $C_{1}$ con otros estados mentales y qué respuestas conductuales sean desencadenadas por él. Análogamente, cabe pensar que podremos identificar puntos de semejanza o parentesco entre dos contenidos diferentes, así como los puntos de divergencia entre ellos, detectando parecidos o diferencias en el tipo de vínculos que estos mantengan con otros estados mentales, con los estímulos y con las respuestas conductuales de la criatura.

Tomando en consideración estas distinciones, podemos ahora señalar algunos de los puntos de semejanza entre el estado mental del mono cuando percibe que allí hay una "fruta" y el que tendríamos nosotros de percibir que allí hay una fruta. En primer lugar, hay semejanzas obvias en el tipo de relaciones funcionales del tipo (i). Las respuestas específicas del mono ante la fruta nos indican que, al menos en buena parte de los casos, el estado perceptual allí hay una "fruta" es desencadenado en él por los mismos tipos de estímulos - las frutas - que suscitan en nosotros estados perceptuales con el contenido allí hay una fruta. En segundo lugar, también es plausible pensar que el presunto estado perceptual de que ahí hay una "fruta" del mono, también produce en él conductas similares a las que muestran los humanos que perciben frutas (es decir que hay similitudes entre los vínculos de tipo iii) de uno y otros). Como nosotros, el mono tiende a arrancar las frutas de los árboles y comerlas, se las da a sus hijos para alimentarlos, etc. Finalmente, aunque sabemos poco sobre los procesos inferenciales de los primates, parece plausible, al menos desde un nivel de explicación folk, atribuir al mono algunos otros estados intencionales inferencialmente vinculados con su estado perceptual acerca de la fruta, de modo análogo a cómo se vinculan entre sí nuestros estados internos correspondientes. Un ejemplo de ello podría ser el del vínculo existente entre la percepción del mono de que la "fruta" está ahí, su deseo de comer fruta, su creencia de que puede arrancar la fruta para comerla, etc. Todos ellos pueden ser entendidos como estados mentales que configuran entre sí una red intencional acotada, a la que apelamos a la hora de explicar y predecir su conducta. ${ }^{10}$

Así pues, ante la pregunta: ¿por qué atribuirle al mono un estado perceptual con 
un contenido como la "fruta" está ahí?, la respuesta es: porque hipotetizamos que la representación de "fruta" que este emplea se asemeja, en algunos aspectos relevantes, a nuestro concepto de fruta. Luego, si se nos sigue preguntando ¿en qué se semejan?, podemos responder: en algunos puntos de su papel funcional. Y, a continuación, podemos pasar a especificar mejor esta respuesta detallando los puntos en los que se superponen, o al menos se asemejan, los papeles funcionales de ambos conceptos y los de los contenidos en los que estos últimos figuran.

\section{El problema de la aspectualidad y la justificación de nuestras atribuciones intencionales a animales no humanos}

Nada de lo que he dicho basta para mostrar, contra Davidson, que somos (o seremos) capaces de atribuir contenidos proposicionales precisos a los animales carentes de lenguaje. Bien puede ocurrir que, aunque empleemos todas las estrategias arriba sugeridas, la ausencia de lenguaje nos deje siempre con evidencia demasiado pobre como para dirimir con total precisión qué piensa una criatura. Puede que lleguemos a saber que ciertas atribuciones son mejores que otras y que esto nos brinde una aproximación parcial a dichos contenidos. Pero también es posible que, dado que los animales no humanos no pueden hablar, haya detalles que se nos escapen ineludiblemente respecto de cómo categorizan el mundo o a qué rasgos de éste responden. Si esto fuera así, seríamos incapaces de capturar con exactitud los contenidos de sus pensamientos. Ahora bien, aún así este fuera el caso, las estrategias esbozadas bastan para concluir que podemos tener conocimiento relevante, aunque quizás sólo aproximado, acerca de los contenidos mentales de los animales y que podemos encontrar modos que resulten mejores que otros para poner en palabras tales contenidos. Habiendo establecido este punto, resta aún dar un paso adicional: argumentar que este conocimiento parcial y tosco basta para justificar la atribución de estados mentales a criaturas sin lenguaje. Esto nos permitirá concluir que, aún si Davidson tiene razón al sostener que, debido a la opacidad referencial de nuestras atribuciones intencionales no podemos hallar atribuciones que capturen de manera precisa los presuntos contenidos mentales de los animales, de allí no se sigue, como él pretende, que tales atribuciones sean injustificadas.

Este último paso argumentativo es relativamente sencillo. Descansa en una reflexión sobre cuán estrictos son nuestros estándares de justificación para atribuir, en contextos ordinarios, creencias a los humanos. Pese a que los animales humanos sí son lingüísticamente competentes, es frecuente que en nuestras interacciones cotidianas con ellos no nos proporcionen el tipo de evidencia conductual rica que necesitamos para determinar con precisión qué tipo de estado mental atribuirles. Diversas razones pueden explicar este hecho: a veces tenemos acceso fragmentario insuficien- 
te a su conducta o, como señalé anteriormente, otras veces la gente presenta patrones conductuales contradictorios, dice ciertas cosas y hace otras, disfraza u oculta sus estados mentales, posee dificultades para comunicarlos, etc. ${ }^{11}$ En todos estos casos la evidencia disponible resulta demasiado pobre, insuficiente o inadecuada para especificar con precisión cuáles son los contenidos mentales de estos sujetos (Wilkes 1997). Esto puede llevarnos a albergar algunas dudas con respecto a qué es exactamente lo que esa persona piensa, o con respecto a si entiende ciertas nociones del mismo modo que nosotros. Sin embargo, por lo general, esto no nos impide continuar atribuyendo a tales agentes estados mentales intencionales, dotados de contenidos aspectuales, con el fin de predecir y explicar su conducta, de comprenderla, cooperar con ella, etc. Más aún, con frecuencia consideramos que tales explicaciones están (al menos relativamente) justificadas. Esto es así pues podemos dar algunas razones para tales atribuciones, aún cuando estas no sean concluyentes (McGinn 1982). Dicho de otro modo: la justificación de nuestras atribuciones no es una cuestión de todo o nada, ni siquiera cuando lidiamos con otros miembros, lingüísticamente competentes, de nuestra propia especie.

Si esto es así cabe preguntarse: ¿por qué no estaríamos justificados de manera análoga a realizar atribuciones mentalistas a los animales carentes de lenguaje, una vez que contemos con evidencia que nos de buenas razones - aunque no razones concluyentes - para atribuirles ciertos contenidos? Consideremos un ejemplo conocido: según revelan las observaciones empíricas, los chimpancés apilan hojas de árboles y luego se sientan sobre ellas como si fueran almohadones (Hirata et al., 1998; McGrew 2004). La evidencia que poseemos puede resultar insuficiente - y en ausencia del lenguaje es posible que no logremos superar este obstáculo - para determinar si dichos chimpancés tienen exactamente el mismo concepto de almohadón que nosotros y, si no lo tienen, en qué puntos difiere su concepto - o su representación - del nuestro. Pero, pese a ello, la evidencia obtenida sí basta para indicarnos que los chimpancés que apilan hojas las representan como teniendo una buena parte de los rasgos relevantes que quedan capturados por nuestro concepto de almohadón. Y son estos puntos en común los que hacen que atribuirle contenidos mentales en los que figure el concepto "almohadón" resulte predictiva y explicativamente útil, al menos en ciertos contextos. Así, por ejemplo, si le atribuimos la intención de construir un almohadón, no nos extrañaremos luego cuando el objeto que construya de hecho sea mullido, ni cuando lo emplee para sentarse sobre él.

En síntesis: a menudo, el conocimiento que podemos reunir acerca del comportamiento y habilidades de los animales parece suficiente para atribuirles pensamientos con contenidos aspectuales, que resulten útiles a la hora de realizar predicciones y explicaciones de su conducta y que capturen - de modo que puede ser impreciso o parcial, pero no completamente inexacto - lo que ellos piensan. En muchos casos, nuestras atribuciones a los animales humanos presentan rasgos similares, pese 
a lo cual seguimos considerando que están justificadas, en la medida en que capturan aspectos relevantes de lo modos en que los agentes se representan el mundo. La evidencia de que una criatura tiene ciertos estados mentales es gradual. Nuestra justificación para atribuirle dichos estados y nuestro conocimiento de los mismos comparten dicho rasgo. Una vez aceptado este punto, el argumento davidsoneano de la opacidad pierde su capacidad para poner en jaque la legitimidad de nuestras atribuciones de estados mentales a los animales, así como también para desalentarnos en nuestros intentos por obtener conocimiento genuino acerca de los mismos.

\section{Referencias}

Bermúdez, J. L. 2003. Thinking without words. Oxford: Oxford University Press.

Block, N. 1986. Advertisement for a Semantics for Psychology. Midwest Studies in Philosophy $\mathrm{X}:$ 615-678.

Carruthers, P. 2004. On being simple-minded. American Philosophical Quarterly 41: 205-220.

Camp, E. 2009. Putting thoughts to work: Concepts, xsistematicity and stimulus-independence. Philosophy and Phenomenological Research 78: 275-311.

Crane, T. 2013. The Objects of Thought. Oxford: Oxford University Press.

Danón, L. 2011. Normatividad doxástica en animales no humanos. Analítica 5: 9-28.

- 2013a. Atribuciones intencionales a animales sin lenguaje: aspectualidad y opacidad referencial. Areté: Revista de Filosofía 3: 54-63.

- 2013b. Conceptos de sustancia y conceptos de propiedades en animales no humanos. Crítica: Revista Hispanoamericana de Filosofía 45: 27-54.

Davidson, D. 1975[1990]. Pensamiento y habla. In: De la verdad y la interpretación, p.164178. Gedisa: Barcelona.

—. 1982[2003]. Animales Racionales. In: Subjetivo, intersubjetivo, objetivo, p.141-155. Madrid: Cátedra.

- 1997. The Emergence of Thought. Erkenntnis 51: 7-17.

Dretske, F. 2000. Perception, Knowledge and Belief. Cambridge: Cambridge University Press.

Duhau, L. 2010. Do Bees Really Have Concepts? A Discussion of Carruthers' Criteria for Conceptuality. Teorema XIX: 125-134.

Glock, H. J. 2000. Animals, thoughts and concepts. Synthese 123: 35-64.

- 2003. Quine and Davidson: on language, thought and reality. Cambridge: Cambridge University Press

Goldman, A. 2006. Simulating Minds: The Philosophy, Psychology and Neuroscience of Mindreading. Oxford: Oxford University Press.

Harman, G. 1973. Thought. New Jersey: Princeton University Press.

Heil, J. 1992. The nature of true minds. Cambridge: Cambridge University Press.

Hurley, S. 2003. Animal Action in the Space of Reasons. Mind and Language 18: 231-256.

Loar, B. 1981. Mind and Meaning. Cambridge: Cambridge University Press.

Lurz, R. 2008. Animal minds. In: Internet Encyclopedia of Philosophy. Disponible online en: http://www.iep.utm.edu/a/ani-mind.htm. 
Lurz, R.; Krachun, C. 2011. How could we know whether nonhuman primates understand other's internal goals and intentions? Solving Povinelli's problem. Review of Philosophy and Psychology 2: 449-481.

Millikan, R. 2004. Varieties of meaning. Cambridge Massachusetts: MIT Press.

McGinn, C. 1982. The Character of Mind. Oxford: Oxford University Press.

Piccinini, G. 2004. Fuctionalism, computationalism and mental states. Canadian Journal of Philosophy 34: 375-410.

Quine, V. W. 1956. Quantifiers and Propositional Attitudes. Journal of Philosophy 53: 177187.

Saidel, E. 2009. Attributing mental representations to animals. In: R. Lurz (ed.) The Philosophy of Animal Minds, p.35-51. Cambridge: Cambridge University Press.

Schwitzgebel, E. 1997. Words About Young Minds: the Concepts of Theory, Representation and Belief in Philosophy and Developmental Psychology. PhD Dissertation, Berkerly Philosophy Department.

- 2001. In between believing. Philosophical Quarterly 51: 76-82.

Searle, J. 1992. The Rediscovery of the Mind. Cambridge Massachusetts: MIT Press.

- 1994. Animal Minds. Midwest Studies in Philosophy 19: 206-219.

- 2006. La mente: una breve introducción. Bogotá: Grupo Editorial Norma.

Sellars. W. 1963. Science, Perception and Reality. Atascadero: Ridgeview Publishing Company.

Stich, S. 1979. Do animals have beliefs? Australasian Journal of Philosophy 57: 15-28.

Tomasello, M.; Carpenter, M.; Call, J.; Behne, T.; Moll, H. 2005. Understanding and sharing intentions: The origins of cultural cognition. The Behavioral and Brain Sciences 28: 675735.

Wilkes, K. 1997. Talking to cats, bats and rats. In: J. Preston (ed.) Thought and Language, Royal Institute of Philosophy Supplement 42. Cambridge: Cambridge University Press.

LAURA DANÓN

Facultad de Filosofía y Humanidades

Facultad de Psicología

Universidad Nacional de Córdoba

ldanon@gmail.com

\section{Notas}

${ }^{1}$ Aunque el objetivo externo pueda ser una buena guía para la identificación de los referentes de los estados conativos de una criatura, en otras ocasiones resulta falible y conduce a errores. Esto es lo que ocurre, por ejemplo, en aquellos casos en los que un agente desea alcanzar un estado de cosas E pero, fallidamente, se comporta de un modo que, de hecho, tiende a la consecución de otro estado de cosas diferentes.

${ }^{2}$ Diversos autores han subrayado la importancia de algunos de estos requisitos como indicadores de intencionalidad. Cfr. Camp (2009), Carruthers (2004), Saidel (2011), Bermúdez (2003), Hurley (2003) y Danón (2011; 2013b). 
${ }^{3}$ Bermúdez (2003) sostiene que el estado de cosas acerca del cual versa un deseo es aquel que, de tener lugar, causaría el cese de dicho deseo. Algo que podemos identificar de modo empírico, añade, cuando el animal deja de realizar la conducta que se veía animada por tal deseo. Sin embargo, él mismo reconoce que hay deseos que pueden no verse satisfechos. Luego, si sólo contáramos con el cese de la conducta relevante como criterio para identificar el referente de los deseos de una criatura, nos veríamos imposibilitados de conocer dicho referente en los casos en los que la satisfacción del deseo no puede producirse, o aún no se ha producido. Mi propuesta pretende estar mejor posicionada para lidiar con tales casos al incorporar dos requisitos - la direccionalidad del comportamiento hacia un objeto o estado de cosas y el cese de la conducta direccionada cuando este objeto o estados de cosas se alcanza - que bastan, cuando se dan independientemente, para proveer cierta evidencia acerca del objeto de deseo y que se refuerzan mutuamente cuando se satisfacen de modo conjunto.

${ }^{4}$ Asumo aquí que hay buenas razones para atribuir al menos algunos conceptos a distintas especies de animales no humanos (Glock 2000; Danón 2013b; Camp 2009). Sin embargo, el punto no es relevante para este trabajo. Quien no comparta esta idea, puede sencillamente reemplazar todas aquellas ocasiones en las que hable de "el referente de los conceptos" de un animal, por "el referente de las representaciones" de un animal, sin que esto afecte al núcleo de la argumentación aquí presentada.

${ }^{5}$ El núcleo de lo expuesto en este sub-apartado b) recupera, de modo abreviado y con ligeras modificaciones, ideas previas presentadas en Danón XXXb.

${ }^{6}$ Desconozco si los sapos son capaces de formar distintas representaciones alternativas de las moscas. Con independencia de ello el punto que quiero marcar continuará siendo válido para cualquier criatura o sistema - real o imaginario - que sólo pudiera representar al referente de sus pensamientos bajo un único aspecto.

${ }^{7}$ La idea aquí es la siguiente: la atribución de un contenido mental determinado a un animal carece de sentido cuando, por todo lo que sabemos acerca de sus habilidades cognitivas y disposiciones conductuales, este es incapaz de pensar en tal contenido, cualesquiera sean sus circunstancias.

${ }^{8}$ Como señala Goldman, este tipo de atribuciones exige emplear el concepto de fruta no sólo de modo indirecto, sino también meta-representacional, pues en este caso nos percatamos explícitamente de que estamos usando nuestro concepto como una representación que trata de expresar, de modo imperfecto, otra representación diferente: aquella que de hecho posee el animal.

${ }^{9}$ Diversas teorías funcionalistas del contenido mental han sido defendidas, entre otros, por Harman (1973), Block (1986) y Loar (1981).

${ }^{10}$ En sentido estricto, el problema de qué conductas animales merecen ser explicadas atribuyéndoles diversos estados mentales vinculados inferencialmente entre sí, merece un debate más extenso, que no puedo abarcar aquí. Numerosos autores coinciden en la necesidad de atribuir algún conjunto acotado de estados mentales interconectados entre sí a fin de explicar diversos comportamientos animales (Hurley 2003; Glock 2000; 2003; Bermúdez 2003; Saidel 2009). Sin embargo, al menos en los casos de comportamientos más simples y rígidos, puede que convenga adoptar una posición diferente. Siguiendo a Millikan (2004) cabe pensar que, en tales ocasiones, el comportamiento animal puede ser explicado de modo más sencillo atribuyendo a los animales un tipo de representaciones primitivas a las que denomina: "representaciones pushmi-pullyu". Estas se componen de dos caras, indisolublemente 
ligadas entre sí: una cara descriptiva, que indica a la criatura cómo es su entorno, y una directiva que le indica cómo ha de actuar en relación con el mismo (un contenido de este tipo podría ser, por ej: “alimento allí/agárralo!"). Luego, al menos cuando la conducta del animal ante la percepción de cierto objeto se desencadene rígidamente, bastará con atribuirle una de estas representaciones básicas para identificar qué es lo que este percibe y para predecir cómo va a actuar al respecto.

${ }^{11}$ He aquí algunos ejemplos conocidos: (i) un niño pequeño afirma que su papá es profesor de filosofía. ¿Cuál es exactamente el contenido de su creencia? ¿Qué entiende él por profesor y por filosofía?; (ii) alguien dice que quiere bajar de peso y afirma estar haciendo dieta, pero sigue comiendo grandes cantidades de grasas y dulces: ¿realmente cree estar haciendo una dieta?; (iii) un artista comienza una pintura de forma vaga e indefinida, dice que quiere representar un bosque, pero termina pintando algo azul que se asemeja a un mar. ¿Qué estaba intentando dibujar?

Principia 20(2): 143-164 (2016). 